\title{
Xenon ventilation MRI in difficult asthma: initial experience in a clinical setting
}

\author{
Grace T. Mussell $\mathbb{1}^{1}$, Helen Marshall $\mathbb{1}^{1}$, Laurie J. Smith $\mathbb{1}^{1}$, Alberto M. Biancardi ${ }^{1}$, Paul J.C. Hughes ${ }^{1}$, \\ David J. Capener ${ }^{1}$, Jody Bray ${ }^{1}$, Andrew J. Swift $\mathbb{D}^{1}$, Smitha Rajaram ${ }^{1}$, Alison M. Condliffe $\mathbb{D}^{2,3}$, \\ Guilhem J. Collier $\mathbb{D}^{1}$, Chris S. Johns $\mathbb{B}^{1}$, Nick D. Weatherley $\mathbb{B}^{1,3}$, Jim M. Wild $\mathbb{D}^{1}$ and Ian Sabroe $\mathbb{B}^{3}$
}

${ }^{1}$ POLARIS, Academic Radiology, Dept of Infection, Immunity and Cardiovascular Disease, University of Sheffield, Sheffield, UK. ${ }^{2}$ Dept of Infection, Immunity and Cardiovascular Disease, University of Sheffield, Sheffield, UK. ${ }^{3}$ Respiratory Medicine, Sheffield Teaching Hospitals, NHS Foundation Trust, Sheffield, UK.

Corresponding author: Ian Sabroe (i.sabroe@nhs.net)

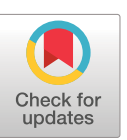

This version is distributed under the terms of the Creative Commons Attribution NonCommercial Licence 4.0. For commercial reproduction rights and permissions contact permissions@ersnet.org

This article has supplementary material available from openres.ersjournals.com

Received: 6 Nov 2020 Accepted: 9 July 2021

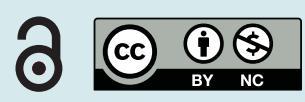

\section{Shareable abstract (@ERSpublications)}

This article demonstrates the feasibility of using ${ }^{129} \mathrm{Xe}$ MRI in clinical practice. Discordance between symptoms, spirometry and MRI can support the use of further treatment or suggest coexisting breathing control issues or laryngeal disorders. https://bit.ly/3ky4oXP

Cite this article as: Mussell GT, Marshall H, Smith LJ, et al. Xenon ventilation MRI in difficult asthma: initial experience in a clinical setting. ERJ Open Res 2021; 7: 00785-2020 [DOI: 10.1183/23120541.007852020].

\section{Abstract}

Background Hyperpolarised gas magnetic resonance imaging (MRI) can be used to assess ventilation patterns. Previous studies have shown the image-derived metric of ventilation defect per cent (VDP) to correlate with forced expiratory volume in $1 \mathrm{~s}\left(\mathrm{FEV}_{1}\right)$ /forced vital capacity (FVC) and $\mathrm{FEV}_{1}$ in asthma. Objectives The aim of this study was to explore the utility of hyperpolarised xenon-129 $\left({ }^{129} \mathrm{Xe}\right)$ ventilation MRI in clinical care and examine its relationship with spirometry and other clinical metrics in people seen in a severe asthma service.

Methods 26 people referred from a severe asthma clinic for MRI scanning were assessed by contemporaneous ${ }^{129} \mathrm{Xe}$ MRI and spirometry. A subgroup of 18 patients also underwent reversibility testing with spirometry and MRI. Quantitative MRI measures of ventilation were calculated, VDP and the ventilation heterogeneity index $\left(\mathrm{VH}_{\mathrm{I}}\right)$, and compared to spirometry, Asthma Control Questionnaire 7 (ACQ7) and blood eosinophil count. Images were reviewed by a multidisciplinary team.

Results VDP and $\mathrm{VH}_{\mathrm{I}}$ correlated with $\mathrm{FEV}_{1}, \mathrm{FEV}_{1} / \mathrm{FVC}$ and forced expiratory flow between $25 \%$ and $75 \%$ of FVC but not with ACQ7 or blood eosinophil count. Discordance of MRI imaging and symptoms and/or pulmonary function tests also occurred, prompting diagnostic re-evaluation in some cases.

Conclusion Hyperpolarised gas MRI provides a complementary method of assessment in people with difficult to manage asthma in a clinical setting. When used as a tool supporting clinical care in a severe asthma service, occurrences of discordance between symptoms, spirometry and MRI scanning indicate how MRI scanning may add to a management pathway.

\section{Introduction}

Asthma management is now based on clinical estimates of severity and pathological phenotypes, focusing at a pathological level on the inflammatory pathways involved. However, clinical practice is often made more challenging by discordance between symptoms and tests such as measures of lung function [1].

Hyperpolarised gas magnetic resonance imaging (MRI) can provide detailed three-dimensional (3D) images of the ventilated lung airspaces [2, 3]. Previous studies on well-characterised cohorts of patients have observed that unventilated regions (ventilation defects) present on MRI are more common in people with asthma than healthy volunteers [4], increase with asthma severity [5-7] and change in response to medications administered to either induce or treat bronchoconstriction [4, 8-11]. Studies have investigated the relationships between MRI metrics, spirometry and clinical measures such as eosinophil count (sputum and/or blood) in patients with asthma [4-7, 10-19], showing moderate correlations of MRI ventilation 
defect per cent (VDP) with forced expiratory volume in $1 \mathrm{~s}\left(\mathrm{FEV}_{1}\right) /$ forced vital capacity (FVC) and $\mathrm{FEV}_{1}$ $[5,7,18,19]$. The relationship between VDP and eosinophil count is less consistent, with some studies reporting a correlation with blood eosinophils and sputum eosinophils [12, 16, 19], and another reporting no correlation with sputum eosinophils [7]. Ventilation defects have also been shown to be associated with clinical outcomes indicative of severe asthma exacerbations such as hospitalisations [19], and to correlate with asthma control in a cohort of people with severe, poorly controlled asthma [15]. Most studies to date have focused on VDP, although some have also considered the heterogeneity within the ventilated regions of the lung $[11,20]$. Relationships between MRI ventilation heterogeneity and lung function have so far not been assessed in people with asthma.

The promise of hyperpolarised gas MRI for the monitoring of lung ventilation is now well established in clinical research studies, where patient populations are strictly defined by inclusion and exclusion criteria, but the ability of the technique to translate into real-world clinical practice is unknown. In 2015 our centre was licenced by the UK regulatory body the Medicines and Healthcare products Regulatory Agency (MHRA) for the manufacture of hyperpolarised ${ }^{129} \mathrm{Xe}$ gas for clinical lung investigations, a world-first for this technology. In this work we therefore aimed to investigate the use of hyperpolarised ${ }^{129} \mathrm{Xe}$ ventilation MRI in a cohort of patients as part of routine care in a difficult asthma service, and its relationship with spirometry and other clinical metrics, and to begin to understand how it might be used to support clinical decision making.

\section{Methods}

\section{Patient referral}

28 adults with difficult-to-manage asthma were referred from the severe asthma service in Sheffield Teaching Hospitals, UK, where difficult-to-manage asthma was taken to include people with severe disease and/or discordance between symptoms and observed tests and signs, and where it was felt that further imaging of lung ventilation may support a diagnostic and management process. The patients studied were referred for MRI between February 14, 2018 and August 28, 2019. Data from the patients were analysed under a study approved by the Yorkshire and the Humber - Sheffield research ethics committee $(17 / \mathrm{YH} /$ 0266). Patients were made aware that the scanning technique was established at a research level and that there was a belief that these scans would complement other investigations in management of their disease, but that its place in clinical practice was under evaluation. They provided written informed consent for the data collected to also be studied to help us examine the utility of MRI and share the knowledge we gained.

\section{MRI acquisition}

28 patients were scanned using a $1.5 \mathrm{~T}$ whole body MRI system (GE HDx, Milwaukee, WI, USA) and a transmit-receive vest coil (CMRS, Brookfield, WI, USA). 3D ventilation images of the lungs were acquired at breath-hold after inhalation of xenon as previously described [21]. ${ }^{129}$ Xe was manufactured on a spin exchange polariser that was regulatory approved for routine clinical usage under a MHRA manufacturing specials regulatory licence (MS-19739). The dose of ${ }^{129} \mathrm{Xe}$ and inhaled volume were titrated based upon patient height. Anatomical ${ }^{1} \mathrm{H}$ images of the same lung volume were also acquired in a separate breath-hold. Further methodological details are described in the supplementary material.

\section{MRI analysis and interpretation}

MRI scans were performed as part of clinical management, with evaluation of scans and correlation with clinical picture within a multidisciplinary team (MDT) comprising at least one radiologist, respiratory clinician and MRI physicist. Image metrics were calculated using semi-automated segmentation [22] for both the ${ }^{1} \mathrm{H}$ and ${ }^{129} \mathrm{Xe}$ images. The ${ }^{129} \mathrm{Xe}$ images were segmented to calculate the ventilated lung volume and the ${ }^{1} \mathrm{H}$ images were used to calculate the total thoracic cavity volume. Using these values the VDP and ventilation heterogeneity index $\left(\mathrm{VH}_{\mathrm{I}}\right)$ [23] were calculated (table 1 and online supplementary material).

In addition to calculation of image metrics, images were reported by radiologists with 11 (A.J.S.), 11 (S.R.) and 7 (C.S.J) years' experience and expertise in chest radiology with input from the MR imaging team of scientists at the MDT. Images were reviewed blind to spirometry data and the radiologists' report was a consensus between two radiologists. Images and radiologists' reports were made available in the clinical radiological imaging system. Radiological reports were based upon a qualitative visual inspection, with minor ventilation abnormality defined as appearing to be $<5 \%$ of lung involvement and substantial ventilation abnormality defined as appearing to be $>5 \%$ of lung involvement. We introduced this terminology in order to capture the qualitative evaluation of experienced radiologists alongside MRI metrics. 


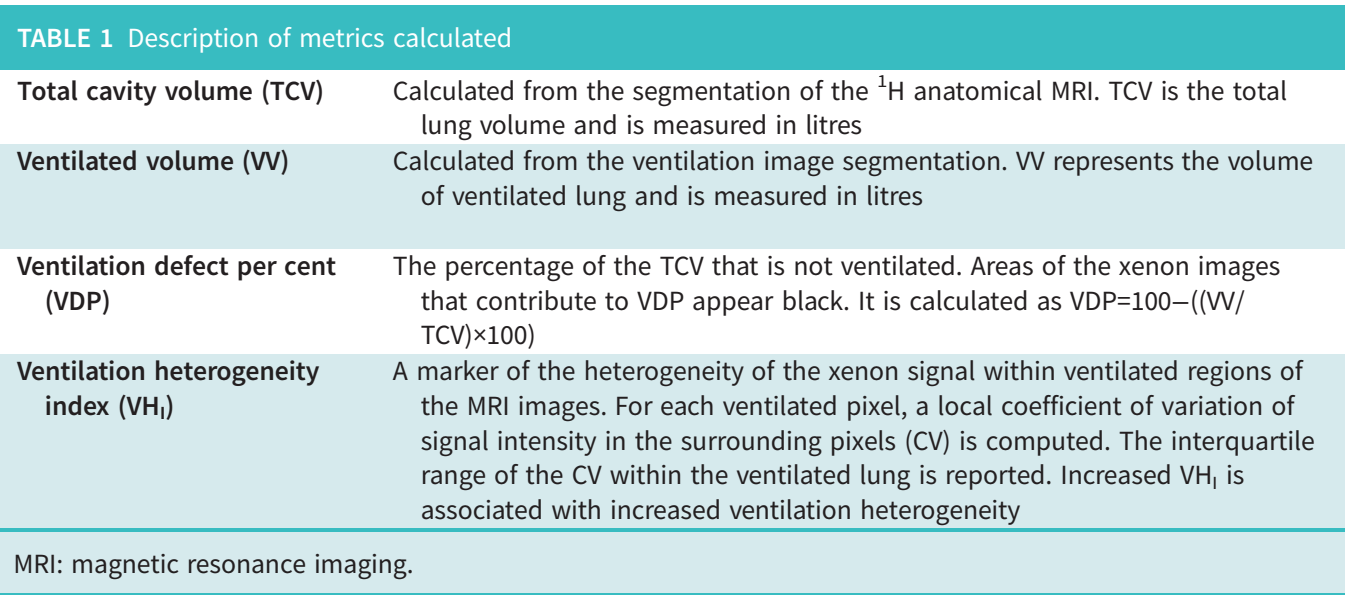

\section{Pulmonary function tests}

Patients underwent spirometry on the day of the MRI scan where possible, conducted in accordance with international guidelines [24]. Three patients did not have spirometry on the day of the scan, and the most recent clinic results were used instead (see supplementary material). $\mathrm{FEV}_{1}, \mathrm{FEV}_{1} / \mathrm{FVC}$, and forced

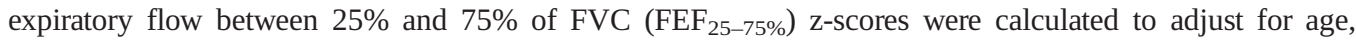
height, sex and ethnicity [25].

\section{Reversibility testing}

Visualisation of bronchodilator reversibility with MRI was requested by the referring clinician in a subset of the cohort (18 patients). Prior to testing patients were advised to withhold all inhalers on the morning of the scan. Where they were receiving long-acting bronchodilators with twice daily dosing, the most recent dose would have been the evening before testing, whereas for once-daily dosing, the most recent dose would have been the morning of the day before testing. Following baseline spirometry and MRI, these patients inhaled $400 \mu \mathrm{g}$ of salbutamol from a metered-dose inhaler via a spacer, while supervised by a physician or physiologist. After $20 \mathrm{~min}$, MRI was repeated followed by repeat spirometry testing. Where patients had taken routine treatment on the morning of the scan and/or reversibility had not been requested, the data were treated as being post-bronchodilator.

\section{Other clinical information}

Relevant demographics, asthma phenotype (eosinophilic non-atopic, eosinophilic-atopic or non-eosinophilic), smoking status, the highest blood eosinophil count within a year of the scan, Asthma Control Questionnaire 7 (ACQ7) score within 3 months of the scan and medications taken around the time of the scan were obtained from clinical records in accordance with the approved protocol.

\section{Statistical analysis}

Data were analysed using GraphPad Prism v7.0 (GraphPad Inc, San Diego, CA, USA). D’Agostino and Pearson normality tests were carried out and the appropriate statistical test or correlation was chosen based upon this. Correlations between MRI metrics, spirometry, ACQ7 and blood eosinophil count were explored using post-bronchodilator data. Where data were not found to be normally distributed, Spearman correlations were used, with Pearson correlations being used if both metrics being assessed for correlation had a normal distribution.

\section{Results}

The MRI scans and spirometry were tolerated well by all patients, and there were no adverse effects reported. 28 scans were potentially eligible for analysis, but one subject was excluded as their scans exhibited poor signal-to-noise ratio and one subject was excluded due to motion artefact in the images.

\section{Patient demographics}

26 patients with asthma were assessed (69\% female). Demographics, spirometry and imaging metrics are given in table 2 .

Spirometry is often interpreted using predicted values, but these run risks of introducing age- and height-related bias [26, 27]. z-scores can be calculated that take into account more variables affecting lung function including age, height, sex and ethnicity, which allows lung function to be compared amongst 
TABLE 2 Subject demographics, lung function and ventilation magnetic resonance imaging metrics

\begin{tabular}{|c|c|c|c|}
\hline & \multirow{2}{*}{$\begin{array}{c}\text { All patients } \\
\text { Post-bronchodilator }\end{array}$} & \multicolumn{2}{|c|}{$\begin{array}{l}\text { Subgroup of those patients in whom } \\
\text { reversibility was assessed }\end{array}$} \\
\hline & & Pre-bronchodilator & Post-bronchodilator \\
\hline Subjects n ( $\%$ female) & $26(69)$ & $18(61)$ & $18(61)$ \\
\hline Age years & $45.7 \pm 12.86$ & $48.8 \pm 12.5$ & $48.8 \pm 12.5$ \\
\hline Height $\mathrm{cm}$ & $166 \pm 8.59$ & $165 \pm 9.00$ & $165 \pm 9.00$ \\
\hline $\mathrm{FEV}_{1} \mathrm{~L}$ & $2.49 \pm 0.82$ & $2.08 \pm 0.88$ & $2.41 \pm 0.86$ \\
\hline $\mathrm{FEV}_{1} \%$ predicted & $79.30 \pm 23.04$ & $65.03 \pm 24.45$ & $75.52 \pm 23.31$ \\
\hline FEV $_{1}$ z-score & $-1.59 \pm 1.55$ & $-2.58 \pm 1.70$ & $-1.84 \pm 1.56$ \\
\hline $\mathrm{FEV}_{1} / \mathrm{FVC} \%$ & $64.27 \pm 14.68$ & $57.53 \pm 13.59$ & $59.63 \pm 13.21$ \\
\hline $\mathrm{FEV}_{1} /$ FVC z-score & $-1.97 \pm 1.44$ & $-2.73 \pm 1.37$ & $-2.35 \pm 1.41$ \\
\hline $\mathrm{FEF}_{25-75 \% \mathrm{~L}}$ & $1.70(5.4)$ & $0.9(2.7)$ & $1.4(2.9)$ \\
\hline $\mathrm{FEF}_{25-75 \%} \%$ predicted & $53.81 \pm 34.30$ & $36.41 \pm 26.22$ & $46.67 \pm 30.07$ \\
\hline $\mathrm{FEF}_{25-75 \%} \mathrm{z}$-score & $-2.96 \pm 1.59$ & $-2.83 \pm 1.41$ & $-2.31 \pm 1.42$ \\
\hline Blood eosinophil count & $0.23(1.10)$ & $0.36(1.10)$ & $0.36(1.10)$ \\
\hline VDP \% & $1.73(28.17)$ & $5.30(31.11)$ & $1.80(28.17)$ \\
\hline $\mathrm{VH}_{\mathrm{I}}$ & $0.13 \pm 0.04$ & $0.14 \pm 0.04$ & $0.13 \pm 0.04$ \\
\hline
\end{tabular}

individuals regardless of these factors [27]. As a result, pulmonary function test z-scores rather than per cent predicted values were used in this analysis. A z-score of -1.64 or greater is considered normal and a z-score of $<-1.64$ is abnormal.

21 subjects had symptoms of inadequately controlled asthma (ACQ7 $\geqslant 1.5$ ) [28], with 16 of these subjects having an ACQ7 >3. Two subjects did not have an ACQ7 score within 3 months of the scan. 25 subjects were taking a long-acting $\beta$-agonist/inhaled corticosteroid combination inhaler; 18 of these were also on a long-acting muscarinic antagonist, 10 were on long-term oral steroids and six on a biologic therapy.

\section{Radiologists' MRI reports}

Eight subjects had no or minor ventilation defects, and 18 patients had substantial ventilation defects ( $>5 \%$ lung involvement). Examples of the variation in ventilation patterns observed in asthma are shown in figure 1. Areas of greater signal intensity (the brighter areas) highlight regions of good ventilation. Ventilation defects are shown as black regions, where there is no signal present due to a lack of ventilation.

Correlations between MRI, spirometry, ACQ7 and blood eosinophil count

VDP, change in $\mathrm{FEV}_{1}$, change in VDP, change in $\mathrm{VH}_{\mathrm{I}}$ and blood eosinophil count failed the normality test, and $\mathrm{VH}_{\mathrm{I}}, \mathrm{FEV}_{1}, \mathrm{FEV}_{1} / \mathrm{FVC}, \mathrm{FEF}_{25-75 \%}$ and ACQ7 passed the normality test. As figure 2 shows,

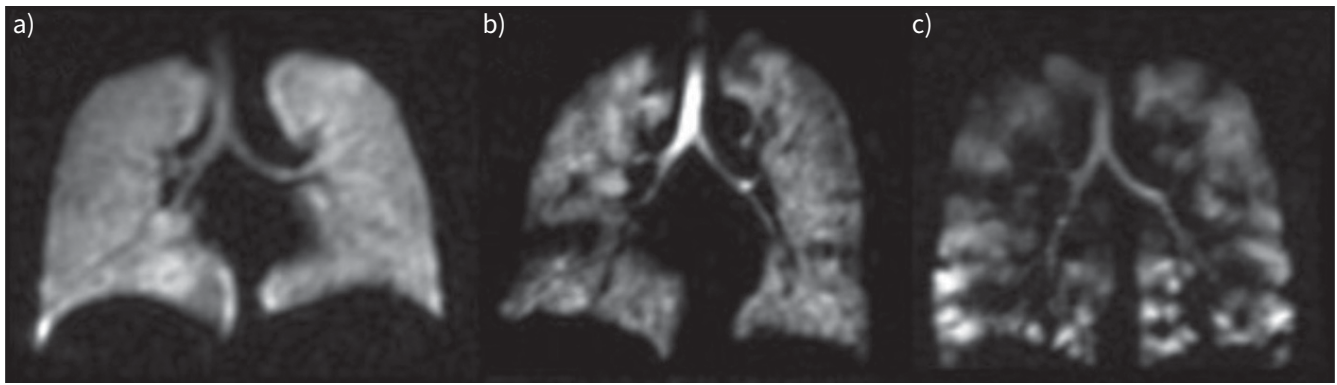

FIGURE 1 Examples of the different visual patterns observed on ventilation magnetic resonance imaging from three patients. Ventilation is shown in greyscale; black regions within the lungs are ventilation defects. a) Subject was reported to have no defects; b) and c) subjects were noted as having substantial ventilation defects on the radiologists' report. a) forced expiratory volume in $1 \mathrm{~s}\left(\mathrm{FEV}_{1}\right)-1.17, \mathrm{FEV}_{1}$ /forced vital capacity (FVC) 0.91 , ventilation defect per cent (VDP) $0.40 \%$, ventilation heterogeneity index $\left(\mathrm{VH}_{1}\right) 0.10$. b) $\mathrm{FEV}_{1}-3.27$, $\mathrm{FEV}_{1} / \mathrm{FVC}-2.99$, VDP $1.85 \%$, $\mathrm{VH}_{1}$ 0.13. c) $\mathrm{FEV}_{1}-2.3, \mathrm{FEV}_{1} / \mathrm{FVC}-3.58$, VDP $17.96 \%, \mathrm{VH}_{1} 0.14$. 


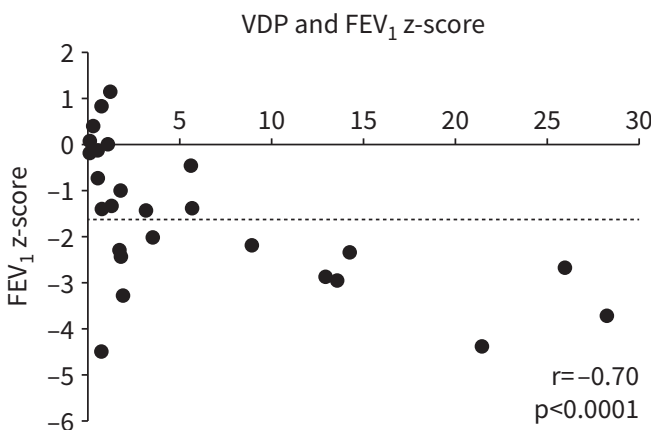

VDP

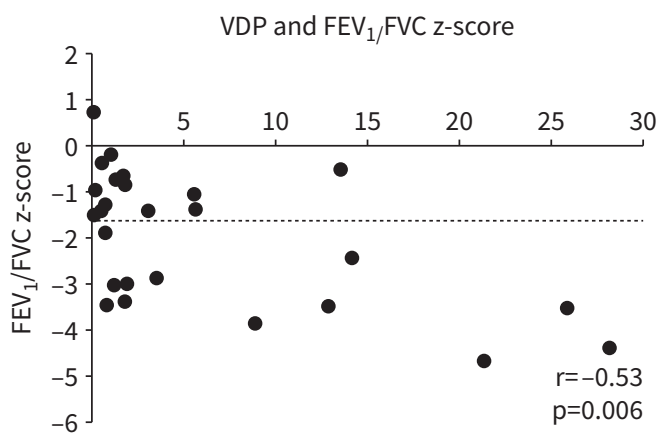

VDP

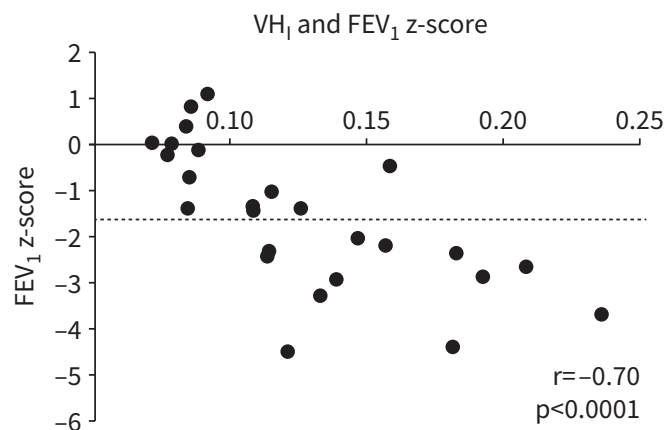

$\mathrm{VH}_{1}$

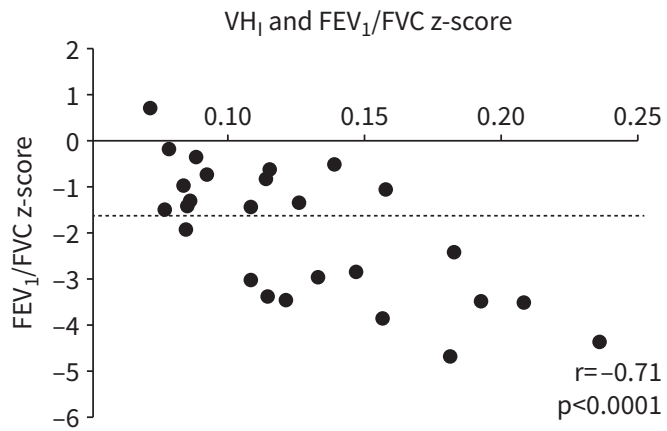

$\mathrm{VH}_{1}$

FIGURE 2 Correlations of ventilation defect per cent (VDP) and ventilation heterogeneity index $\left(\mathrm{VH}_{1}\right)$ with forced expiratory volume in $1 \mathrm{~s}\left(\mathrm{FEV}_{1}\right)$ and $\mathrm{FEV}_{1} /$ forced vital capacity (FVC). Data show the correlations in VDP and $\mathrm{FEV}_{1}, \mathrm{VH}_{1}$ and $\mathrm{FEV}_{1}, \mathrm{VDP}$ and $\mathrm{FEV}_{1} / \mathrm{FVC}$, and $\mathrm{VH}_{1}$ and $\mathrm{FEV}_{1} / \mathrm{FVC}$. The lower limit of normal is indicated at -1.64 z-scores. Spearman correlations with VDP and Pearson correlations with $\mathrm{VH}_{\mathrm{l}}$.

across the studied cohort there were correlations between VDP and $\mathrm{FEV}_{1}(\mathrm{r}=-0.70, \mathrm{p}<0.0001)$ and VDP and $\mathrm{FEV}_{1} / \mathrm{FVC}(\mathrm{r}=-0.53, \mathrm{p}=0.006)$. There were strong correlations between $\mathrm{VH}_{\mathrm{I}}$ and $\mathrm{FEV}_{1}(\mathrm{r}=-0.70$, $\mathrm{p}<0.0001), \mathrm{VH}_{\mathrm{I}}$ and $\mathrm{FEV}_{1} / \mathrm{FVC}(\mathrm{r}=-0.71, \mathrm{p}<0.0001)$. Not illustrated in figure 2, there were also strong correlations with VDP and $\mathrm{FEF}_{25-75 \%}(\mathrm{r}=-0.60, \mathrm{p}=0.002)$ and $\mathrm{VH}_{\mathrm{I}}$ and $\mathrm{FEF}_{25-75 \%}(\mathrm{r}=-0.68, \mathrm{p}=0.0004)$. VDP had a strong correlation with $\mathrm{VH}_{\mathrm{I}}(\mathrm{r}=0.93$, $\mathrm{p}<0.0001)$.

An ACQ7 score was available for 24 patients from within 3 months of the scan. Mean ACQ7 score was 2.96 and ACQ7 did not show correlations with VDP or $\mathrm{VH}_{\mathrm{I}}$. ACQ7 correlated with $\mathrm{FEV}_{1}(\mathrm{r}=-0.51, \mathrm{p}=0.012)$ and $\mathrm{FEF}_{25-75 \%}\left(\mathrm{r}=-0.50, \mathrm{p}=0.02\right.$ ). There was no correlation between ACQ7 and $\mathrm{FEV}_{1} / \mathrm{FVC}$. There were no correlations between blood eosinophil count and MRI metrics or spirometry (data not shown).

\section{Reversibility testing}

18 subjects were tested for reversible airways obstruction. 10 subjects had reversible airways obstruction defined as an improvement in $\mathrm{FEV}_{1}$ of $>12 \%$ and an absolute increase in value of at least $200 \mathrm{~mL}$ [29]. There were significant differences in $\mathrm{FEV}_{1}(\mathrm{p}<0.0001)$, VDP $(\mathrm{p}=0.0004)$ and $\mathrm{VH}_{\mathrm{I}}(\mathrm{p}=0.0217)$ following bronchodilator administration. There were no significant correlations between the change in $\mathrm{FEV}_{1}$ and the change in VDP or $\mathrm{VH}_{\mathrm{I}}$ following bronchodilator.

By qualitative visual assessment, eight patients showed improvement in MRI ventilation following bronchodilator, while 10 subjects did not. Detail regarding the subjects' reversibility judged by both their $\mathrm{FEV}_{1}$ and visual changes on their MRI scans is shown in table $3 . \mathrm{FEV}_{1}$ reversibility testing and qualitative review of MRI were in agreement for 12 patients. Ventilation improvement on MRI was reported for two patients without $\mathrm{FEV}_{1}$ reversibility, and no ventilation improvement on MRI was reported for four patients who showed $\mathrm{FEV}_{1}$ reversibility.

Examples of MRI reversibility images are shown in figure 3. The subject shown in panel A showed effective reversibility following bronchodilator, in both their ventilation MRI scan (VDP reduction of 9.7\%) and in their $\mathrm{FEV}_{1}$ (48\% increase). The subject in panel B showed an apparent deterioration in their 
TABLE 3 Summary of subjects' reversibility in their $\mathrm{FEV}_{1}$, magnetic resonance imaging metrics and visual changes in response to bronchodilator

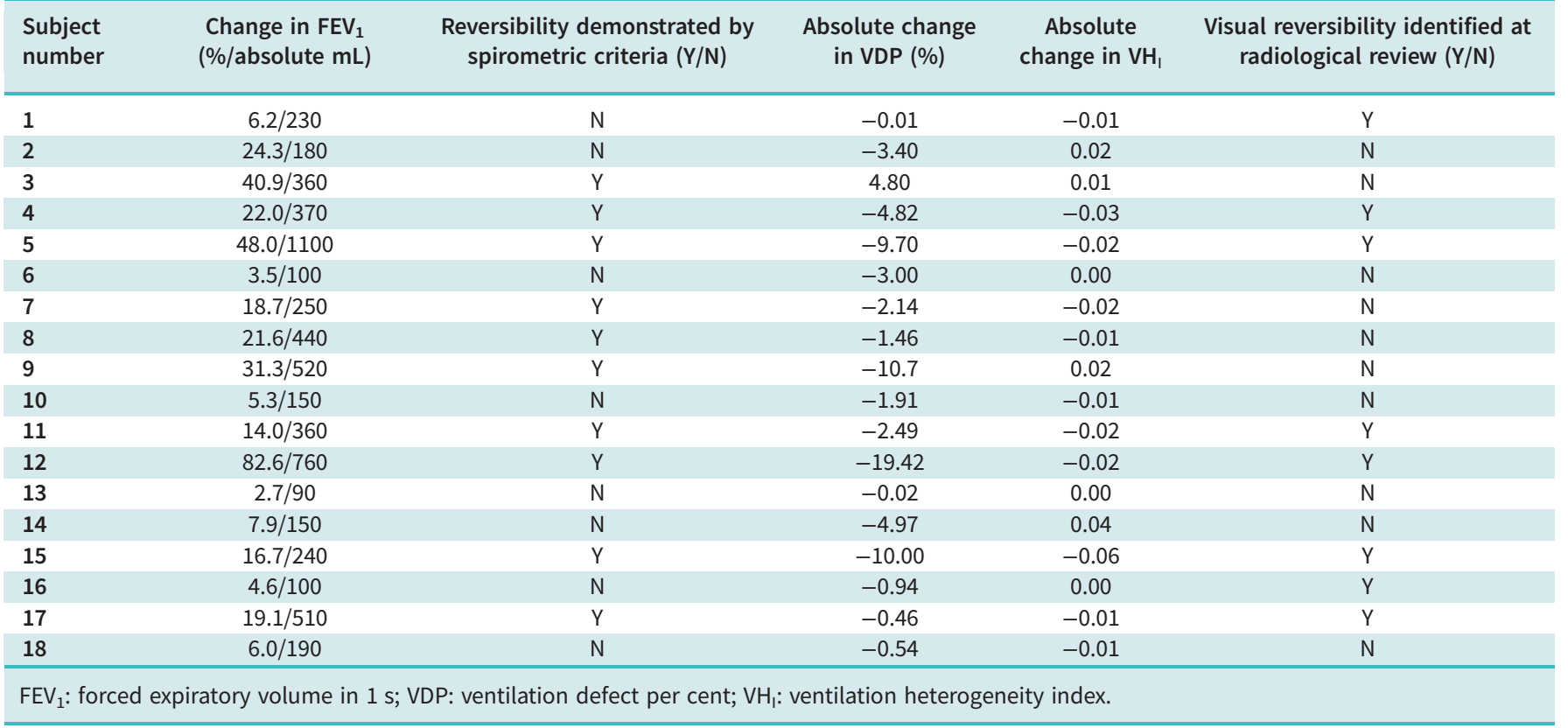

ventilation on MRI (VDP increase of 4.8\%), yet despite this showed some reversibility in their $\mathrm{FEV}_{1}$ (40.9\% increase).

\section{Contribution of MRI to clinical assessment}

Of those in whom spirometry and MRI were performed on the same day, four patients had either an FEV 1 z-score within the normal range $(>-1.64)$ and normal range $\mathrm{FEV}_{1} / \mathrm{FVC}$ z-score values of $>-1.64$. A clinical diagnosis of asthma was made in all of these individuals. Owing to the stochastic nature of asthma, previous spirometry results obtained from these patient records ensured that they had a history of normal results. Substantial ventilation defects on MRI were present in all (table 4). One subject (figure 4a) had consistently normal spirometry by z-score and per cent predicted values over several clinical reviews, yet had a peripheral blood eosinophilia, recurrent symptomatic exacerbations and wheeze, a significant smoking history, and was on maximum standard inhaled therapies. A ventilation MRI scan performed to assist in evaluating their disease showed small to moderate sized ventilation defects with poor reversibility. The patient was commenced on mepolizumab, after 3 months of which they described a transformation of their symptoms and had experienced no significant exacerbations.

In contrast, there were subjects with abnormal spirometry and a high symptom burden who had better than expected ventilation on MRI. Seven patients with abnormal ACQ7 were reported as having no or minor ventilation defects on MRI (table 4).

In other subjects, defects were not as prominent as had been anticipated, which prompted re-evaluation of dysfunctional breathing. An example of this is shown in figure 4b. (This patient was scanned after the data analysis period, and therefore their results are not incorporated into the analyses above.) The individual was highly symptomatic with blood eosinophilia, on long-term oral steroids and had failed to respond to treatment with a biologic. The MRI scan showed minor changes, which prompted re-evaluation for vocal cord dysfunction.

\section{Discussion}

In this study we present a first exploration of the use of ventilation MRI for the benefit of people with asthma in a clinical setting. These analyses not only confirm that relationships already explored in research studies in asthma between VDP and spirometry exist in a real-world clinical cohort, but also show that MRI scanning may assist in the evaluation and management of people presenting with discordance between symptoms and lung function.

There were diverse patterns of ventilation heterogeneity observed in this population of patients, with defects that mechanistically could encompass both large or small airways components, or mixes of the two. 

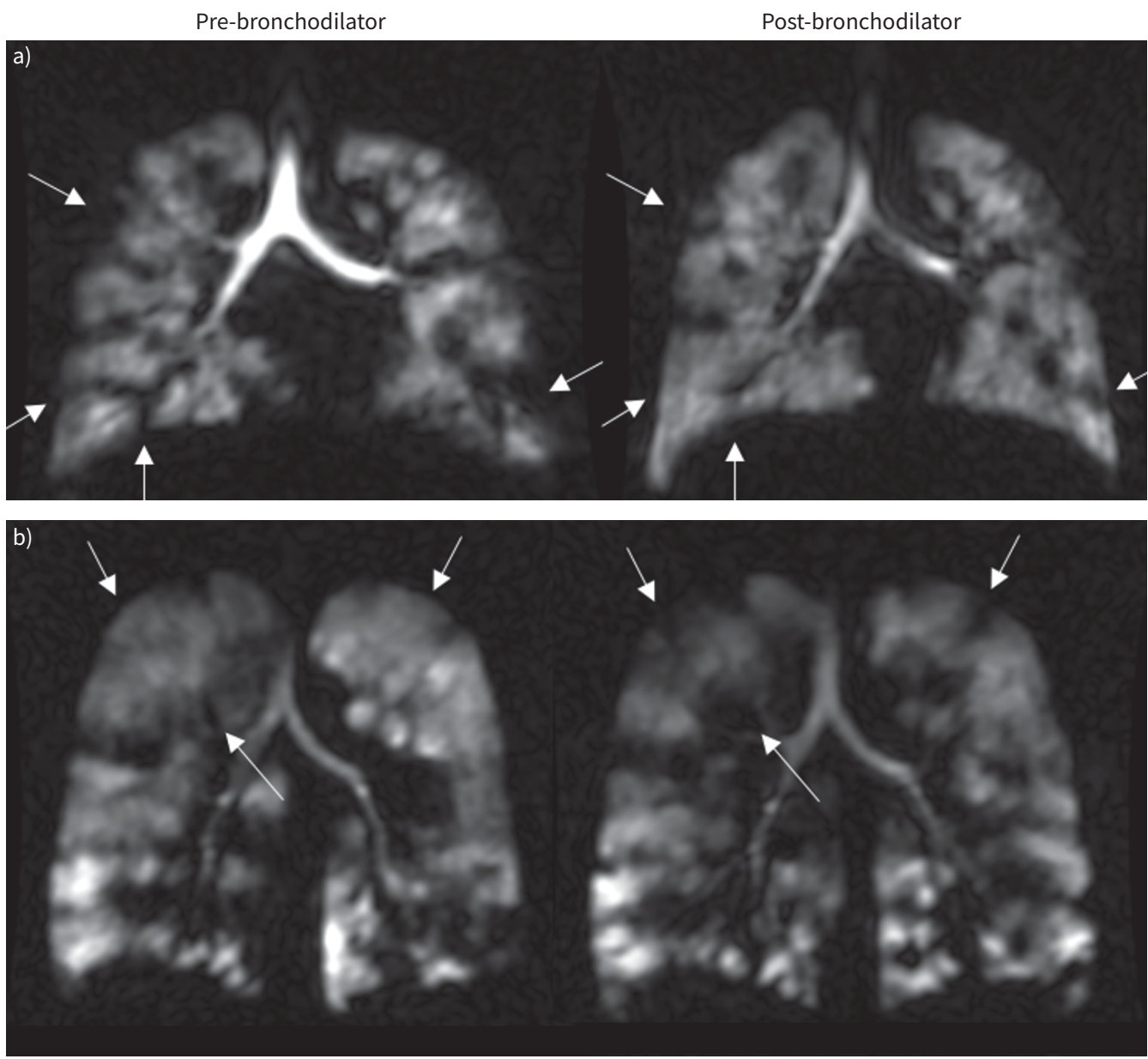

FIGURE 3 Images showing changes with bronchodilator in two different patients: a) subject 5 and b) subject 3 . Arrows highlight areas where defects resolved or worsened. Images show increased ventilation in subject in a) after bronchodilator inhalation and decreased ventilation in subject in b). Table 3 shows details of each subject's metrics.

Defining an accurate clinical reporting terminology of these images will likely require consensus-building work with systematic qualitative radiological interpretation of the regional ventilation heterogeneity seen. In terms of clinical reporting, we believe it is important to report the VDP and $\mathrm{VH}_{\mathrm{I}}$ as unsubjective, quantitative metrics and, for clinical utility, to ensure that images are interpreted within an MDT that includes radiologists, imaging scientists and clinicians who build up experience with the images and metrics.

The apparent 5\% of lung involvement cut-off used for radiological reporting of ventilation MRI was a qualitative assessment chosen by consensus of three experienced chest radiologists with input from the imaging physicists to reflect the threshold we perceived to differentiate between minor and substantial

TABLE 4 Summary of subjects' MRI classification, spirometry and ACQ7 scores

\begin{tabular}{|c|c|c|c|c|c|c|}
\hline \multirow[t]{2}{*}{ MRI classification } & \multicolumn{2}{|c|}{ FEV $_{1}$} & \multicolumn{2}{|c|}{$\mathrm{FEV}_{1} / \mathrm{FVC}$} & \multicolumn{2}{|r|}{ ACQ7 } \\
\hline & $\begin{array}{c}\text { Normal } \\
(>-1.64)\end{array}$ & $\begin{array}{l}\text { Abnormal } \\
(\leqslant-1.64)\end{array}$ & $\begin{array}{l}\text { Normal } \\
(>-1.64)\end{array}$ & $\begin{array}{l}\text { Abnormal } \\
(\leqslant-1.64)\end{array}$ & $\begin{array}{c}\text { Controlled } \\
(<1.5)\end{array}$ & $\begin{array}{l}\text { Inadequately controlled } \\
\qquad(\geqslant 1.5)\end{array}$ \\
\hline Normal & 8 & 1 & 8 & 1 & 2 & 7 \\
\hline Abnormal & 6 & 11 & 5 & 12 & 1 & 14 \\
\hline
\end{tabular}

Magnetic resonance imaging (MRI) classified based on radiologists' reports (normal: no or minor ventilation defects; abnormal: substantial ventilation defects). ACQ7: Asthma Control Questionnaire 7; FEV ${ }_{1}$ forced expiratory volume in $1 \mathrm{~s}$; FVC: forced vital capacity. 

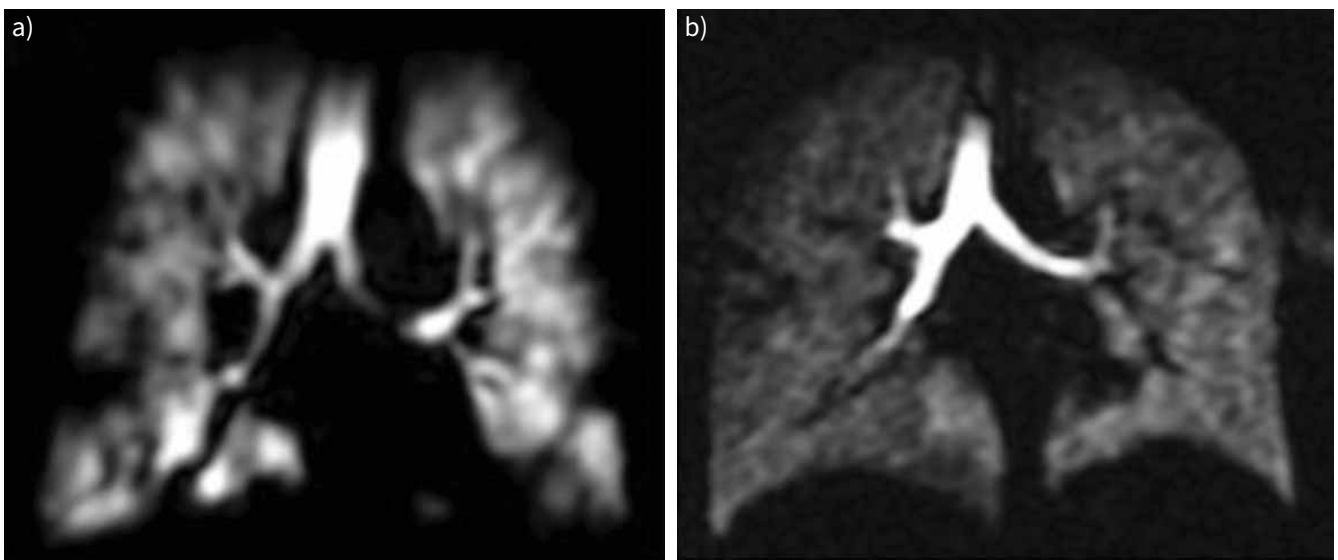

FIGURE 4 Contribution of magnetic resonance imaging to clinical assessment. a) Small to moderate sized ventilation defects in a symptomatic patient with consistently normal spirometry. b) Relatively homogeneous ventilation in a highly symptomatic patient.

ventilation abnormality. We included this parallel approach as a way of generating clinical reports of the images within the context of an MDT evaluation, in which respiratory clinicians, experienced radiologists and MRI physicists examined the scans and considered the images, MRI metrics and clinical history together to reach a best interpretation and application of the images to clinical care. Although an entirely qualitative overview, this " $5 \%$ of lung involvement cut-off" is similar to the VDP threshold of $4.28 \%$ recently found to separate patients who had a median of 1.5 exacerbations in the previous year (VDP $>4.28 \%$ ) from those with a median of 0 exacerbations (VDP $<4.28 \%$ ) in a group of 67 adults and children with a range of asthma severity [30]. EDDY et al. [31] when assessing the minimal clinically important difference (MCID), the smallest measurement difference that patients perceive as beneficial, found the VDP MCID to be 4\% using ACQ score as an anchor in a group of 18 patients with poorly controlled severe asthma.

Correlations between MRI, spirometry, ACQ7 and blood eosinophil count

Significant correlations between ventilation defects measured by MRI and FEV $/ F V C$ and $\mathrm{FEV}_{1}$ have been shown using ${ }^{3} \mathrm{He}[5,18,19]$ and ${ }^{129} \mathrm{Xe}$ [7] in previous research studies. Consistent with these reports, we likewise observed correlations between ${ }^{129} \mathrm{Xe} \mathrm{VDP}$ and $\mathrm{FEV}_{1}, \mathrm{FEV}_{1} / \mathrm{FVC}$ and $\mathrm{FEF}_{25-75 \%}$. These values continue to support VDP as a good metric of airways obstruction in asthma. We report the first observations of correlations between $\mathrm{VH}_{\mathrm{I}}$ and spirometry, which were similar or stronger than correlations of VDP with spirometry. Measures of ventilation heterogeneity provide quantitative information about the uniformity of ventilation within the functional airspaces, which is complementary to VDP.

Correlations between VDP and the presence of eosinophils (blood and sputum) have been shown in some [12, 16, 19] but not all [7] studies. We did not observe correlations between VDP or $\mathrm{VH}_{\mathrm{I}}$ and highest blood eosinophil counts in the last 12 months; however, same-day blood eosinophil levels were not obtained on the day of scanning. A previous recent study found no correlation between ventilation defects on ${ }^{129}$ Xe MRI and asthma control [7]. Similarly in our cohort, symptom burden and ventilation metrics did not show significant correlations, highlighting the known propensity for asthma to exhibit discordant patterns of disease, as previously identified in cluster analysis [1].

\section{Discordance between MRI and spirometry}

Spirometric and MRI changes after bronchodilator inhalation did not correlate. Improvements in MRI ventilation post-bronchodilator have been previously shown to be independent of $\mathrm{FEV}_{1}$ reversibility [32, 33]; however, why this should be remains an understudied area. Inhaled gas ventilation MRI is performed differently to forced flow-based spirometry. MRI-based reversibility is measured as a change in ventilated volume of the lungs at a static point after an inhaled volume of gas that is below that of a maximal inspiration (similar to end-inspiratory tidal volume), performed supine. In contrast, spirometric reversibility is measured in the context of a maximal forced manoeuvre, performed upright. Airways will therefore be subject to different forces, from altered respiratory mechanics of lying flat, and different levels of stretch on airways from lung tissue at different inflation volumes. The maximal inspiration of a spirometric test 
should allow greatest opportunities for reversibility to be demonstrated. However, it is conceivable that airway inflammation and damage may leave airways prone to rapid collapse during forced manoeuvres that can to some degree obscure reversibility that could be seen in MRI-based measures. Furthermore, regional changes in MRI ventilation may occur without a change in spirometry that reaches a diagnostic threshold, particularly since MR images can be examined regionally/anatomically. Thus, small defects typical of airway disease may be visible on an MRI but not seen in terms of reducing a spirometric value sufficiently to render it definitely abnormal. In keeping with these concepts, changes in measured overall VDP, which again averages the whole lung rather than allowing a region-by-region or defect-by-defect view of obstruction, did not always correlate with visual assessment of reversibility. Our current practice is to assess spirometric reversibility and MRI reversibility at the same visit, and it seems clear that these two parallel assessments both contribute to understanding the dynamic changes on the airways imposed by asthma and its treatment with bronchodilators.

In this study four subjects with normal spirometry had evidence of airways obstruction on MRI. DE LANGE et al. [5] also found that more people with asthma had increased ventilation defects with normal spirometry than the converse. This is supported by work in children with cystic fibrosis which has shown that ventilation MRI is more sensitive to early lung disease than spirometry [34, 35].

In the context of discordance between traditional tests and symptoms, MRI offers two opportunities to shape the therapeutic discussion: (1) confirmation of disease when tests or the clinical picture is not clear cut and (2) identification of people with a high symptom burden and relatively normal lung ventilation, in whom the search for additional diagnosis or other ways to help control their breathlessness such as physiotherapy for dysfunctional breathing or consideration of inducible laryngeal obstruction may be valuable and can be reappraised.

\section{Limitations}

There are several limitations to this study which require consideration, many of them related to the clinical nature of the study and the patient referral pathway. While we reported a sample size on a par with those previously reported for hyperpolarised gas MRI studies [11, 16, 36], it still remains a small sample for clinical research. Patients were selected on the basis of a clinical opinion that regional visualisation and quantitative measurement of airway function would complement other modalities of routine assessment, thus this study cannot be generalised to make assumptions about the proportions of MRI scans that would or would not show abnormalities had all people in our service with a diagnosis of severe asthma (however defined) or problematic breathlessness been scanned. Two subjects were excluded on the basis of poor quality in their MRI scans. One of these was due to low image quality caused by a fault resulting in a one-off increase in noise in the system. The other scan was excluded due to motion artefact, likely caused by the patient struggling with the breath-hold. For three subjects, spirometry and the MRI scan were not performed on the same day due to pulmonary function laboratory availability; therefore, the spirometry might not give an accurate representation of how the person was on the day of the scan in these cases. However, other MRI studies have identified that a substantial proportion of ventilation defects in people with asthma show significant stability over time [13].

This was our first experience of using MRI imaging for asthma in a non-research setting in people with often significant breathlessness. In order to reduce a perceived risk of subjects becoming breathless during the scan, we did not ask people to withhold bronchodilators for as long as most recent guidelines [37] recommend. However, scans were well tolerated, and in the future, we would more confidently follow standard guidelines for withholding long-acting bronchodilators pre-testing. Thus we may have underestimated some reversibility in our population, but this would likely apply equally to both MRI and spirometric assessments and is unlikely to have substantially influenced our results. We did not obtain ACQ5 or ACQ6 scores, blood eosinophil counts or other measures of allergic airway inflammation such as exhaled nitric oxide contemporaneously with the MRI scan and did not build in a challenge test to assess bronchial hyper-reactivity (which can be incorporated in MRI protocols [10]); thus we have not attempted to distinguish pathological mechanisms of airway obstruction. Rather, we have focused on the utility of MRI to help understand a clinical picture of breathlessness. In the future, better measures of airway inflammation around the time of MRI scanning may provide additional data that help the interpretation of these scans in clinical practice. We did not exclude patients from MRI scans who had received recent oral corticosteroids. We therefore did not report the temporally closest blood eosinophil count to the scan, as these may have been biased by recent treatment changes and be less representative of typical values; we have provided highest blood eosinophil count in the last year to indicate the proportion of people likely to have eosinophilic inflammation. 
Not every centre is able to undertake tests such as induced sputum eosinophil measurements (particularly during the current coronavirus disease 2019 (COVID-19) pandemic when such aerosol-generating procedures are likely to be unfeasible for some time to come), so future evaluations may focus on contemporaneous blood eosinophil count, highest eosinophil counts in recent history and measures of exhaled nitric oxide.

\section{Conclusion}

This article reports on the use of ${ }^{129}$ Xe ventilation MRI as part of clinical practice for the first time. The ventilation MRI metrics of VDP and $\mathrm{VH}_{\mathrm{I}}$ correlated strongly with spirometry in a real-world clinical cohort of people referred from a severe asthma service. However, of particular interest were those subjects in whom discordance existed between MRI metrics and other features and measures of disease severity. Evidence of airways obstruction on MRI can support the use of further treatment in patients where the clinical picture is unclear, for example those with normal spirometry and high symptom burden. Alternatively, well-preserved ventilation on MRI alongside poor spirometry and/or symptom control may suggest the possibility of coexisting breathing control issues or laryngeal disorders.

Asthma is notorious as a disease for its episodic nature, paroxysmal dangerous exacerbations, complex relationship between symptoms, signs and physical deconditioning, mental distress, and complicating diagnoses such as vocal cord disorders. We have shown that ${ }^{129}$ Xe MRI can provide additional unique and valuable information in the evaluation of clinical presentations of asthma, when undertaken as part of an MDT evaluation of severe disease. Where feasible, it is likely that ventilation MRI would add a useful dimension of assessment that could reasonably be applied to the majority of people with difficult asthma in order to provide additional diagnostic insights. A robust protocol would collect spirometry, symptom control data, measures of inflammation (exhaled nitric oxide, blood eosinophil count) and either reversibility or bronchial hyper-reactivity challenge in parallel with the MRI images. It may be especially valuable in those in whom there is discordance between symptoms, lung function and treatment response, but centres using this technology will need to build experience incorporating it alongside a full MDT assessment. Future studies and ongoing service evaluations will be needed to continue to explore the use of this valuable new imaging technique within assessment protocols of the breathless patient and the patient with severe asthma. The opportunity to show people with breathlessness the images of their lung ventilation may generate new ways to communicate important knowledge about their disease, and the value of images to support and convey health education would be an important qualitative study.

Provenance: Submitted article, peer reviewed.

Acknowledgements: Thanks to Graham Norquay and Oliver Rodgers (polariser operation/maintenance), Madhwesha Rao (RF coil maintenance), Jenny Rodgers and Leanne Armstrong (patient scheduling and administration), and Martin Brook (information technology infrastructure) (POLARIS, Academic Radiology, Dept of Infection, Immunity and Cardiovascular Disease, University of Sheffield, Sheffield, UK).

Conflict of interest: AstraZeneca awarded a research grant to I. Sabroe, J.M. Wild, H. Marshall and colleagues for the period 2020-2023 to study effects of therapies on airway imaging. In 2019, I. Sabroe participated in a scientific advisory board for AstraZeneca, generating a small consultancy income paid to the University of Sheffield to support research, and has attended an annual respiratory research meeting supported by Boehringer Ingelheim. J.M. Wild and H. Marshall attended and advised on an AstraZeneca global respiratory experts meeting, generating a small consultancy income paid to the University. All other authors declare no conflicts of interest related to this study.

Support statement: This work was funded by the Medical Research Council (grant number MR/M008894/1). Funding information for this article has been deposited with the Crossref Funder Registry.

\section{References}

1 Haldar P, Pavord ID, Shaw DE, et al. Cluster analysis and clinical asthma phenotypes. Am J Respir Crit Care Med 2008; 178: 218-224.

2 Salerno M, Altes TA, Mugler JP, et al. Hyperpolarized noble gas MR imaging of the lung: potential clinical applications. Eur J Radiol 2001; 40: 33-44.

3 Castro M, Fain SB, Hoffman EA, et al. Lung imaging in asthmatic patients: the picture is clearer. J Allergy Clin Immunol 2011; 128: 467-478.

4 Altes TA, Powers PL, Knight-Scott J, et al. Hyperpolarized 3He MR lung ventilation imaging in asthmatics: preliminary findings. J Magn Reson Imaging 2001; 13: 378-384. 
5 de Lange EE, Altes TA, Patrie JT, et al. Evaluation of asthma with hyperpolarized Helium-3 MRI. Chest 2006; 130: 1055-1062.

6 Zha W, Kruger SJ, Cadman RV, et al. Regional heterogeneity of lobar ventilation in asthma using hyperpolarized Helium-3 MRI. Acad Radiol 2018; 25: 169-178.

7 Ebner L, He M, Virgincar RS, et al. Hyperpolarized 129Xenon magnetic resonance imaging to quantify regional ventilation differences in mild to moderate asthma: a prospective comparison between semiautomated ventilation defect percentage calculation and pulmonary function tests. Invest Radiol 2017; 52: 120-127.

8 Samee S, Altes TA, Powers P, et al. Imaging the lungs in asthmatic patients by using hyperpolarized helium-3 magnetic resonance: assessment of response to methacholine and exercise challenge. J Allergy Clin Immunol 2003; 111: 1205-1211.

9 Costella S, Kirby M, Maksym GN, et al. Regional pulmonary response to a methacholine challenge using hyperpolarized 3He magnetic resonance imaging. Respirology 2012; 17: 1237-1246.

10 de Lange EE, Altes TA, Patrie JT, et al. The variability of regional airflow obstruction within the lungs of patients with asthma: assessment with hyperpolarized helium-3 magnetic resonance imaging. J Allergy Clin Immunol 2007; 119: 1072-1078.

11 Svenningsen S, Kirby M, Starr D, et al. Hyperpolarized 3He and 129Xe MRI: differences in asthma before bronchodilation. J Magn Reson Imaging 2013; 38: 1521-1530.

12 Altes TA, Mugler lii JP, Ruppert K, et al. Clinical correlates of lung ventilation defects in asthmatic children. J Allergy Clin Immunol 2015; 137: 789-796.

13 de Lange EE, Altes TA, Patrie JT, et al. Changes in regional airflow obstruction over time in the lungs of patients with asthma: evaluation with He-3 MR imaging. Radiology 2009; 250: 567-575.

14 Svenningsen S, Kirby M, Starr D, et al. What are ventilation defects in asthma? Thorax 2014; 69: 63-71.

15 Svenningsen S, Nair P, Guo F, et al. Is ventilation heterogeneity related to asthma control? Eur Respir J 2016; 48: $370-379$

16 Svenningsen S, Eddy RL, Lim HF, et al. Sputum eosinophilia and magnetic resonance imaging ventilation heterogeneity in severe asthma. Am J Respir Crit Care Med 2018; 197: 876-884.

17 Horn FC, Marshall H, Collier GJ, et al. Regional ventilation changes in the lung: treatment response mapping by using hyperpolarized gas MR imaging as a quantitative biomarker. Radiology 2017; 284: 854-861.

18 Capaldi DPI, Eddy RL, Svenningsen S, et al. Free-breathing pulmonary MR imaging to quantify regional ventilation. Radiology 2018; 287: 693-704.

19 Mummy DG, Kruger SJ, Zha W, et al. Ventilation defect percent in helium-3 magnetic resonance imaging as a biomarker of severe outcomes in asthma. J Allergy Clin Immunol 2018; 141: 1140-1141.

20 Tzeng Y-S, Lutchen K, Albert M. The difference in ventilation heterogeneity between asthmatic and healthy subjects quantified using hyperpolarized 3 He MRI. J Appl Physiol (1985) 2008; 106: 813-822.

21 Stewart NJ, Norquay G, Griffiths PD, et al. Feasibility of human lung ventilation imaging using highly polarized naturally abundant xenon and optimized three-dimensional steady-state free precession. Magn Reson Med 2015; 74: 346-352.

22 Hughes PJC, Horn FC, Collier GJ, et al. Spatial fuzzy c-means thresholding for semiautomated calculation of percentage lung ventilated volume from hyperpolarized gas and ${ }^{1} \mathrm{H}$ MRI. J Magn Reson Imaging 2018; 47: 640-646.

23 Hughes PJC, Smith L, Chan H-F, et al. Assessment of the influence of lung inflation state on the quantitative parameters derived from hyperpolarized gas lung ventilation MRI in healthy volunteers. J Appl Physiol (1985) 2019; 126: 183-192.

24 Miller MR, Hankinson J, Brusasco V, et al. Standardisation of spirometry. Eur Respir J 2005; 26: 319-338.

25 Quanjer PH, Stanojevic S, Cole TJ, et al. Multi-ethnic reference values for spirometry for the 3-95-yr age range: the global lung function 2012 equations. Eur Respir J 2012; 40: 1324-1343.

26 Quanjer PH, Enright PL, Miller MR, et al. The need to change the method for defining mild airway obstruction. Eur Respir J 2011; 37: 720-722.

27 Stanojevic S, Quanjer P, Miller MR, et al. The Global Lung Function Initiative: dispelling some myths of lung function test interpretation. Breathe 2013; 9: 462-474.

28 Juniper EF, Bousquet J, Abetz L, et al. Identifying 'well-controlled' and 'not well-controlled' asthma using the Asthma Control Questionnaire. Respir Med 2006; 100: 616-621.

29 National Institute for Health and Care Excellence. Asthma: diagnosis, monitoring and chronic asthma management (NICE Guideline NG80). 2017. www.nice.org.uk/guidance/ng80 Date last accessed: 22 March 2021.

30 Mummy DG, Carey KJ, Evans MD, et al. Ventilation defects on hyperpolarized helium-3 MRI in asthma are predictive of 2-year exacerbation frequency. J Allergy Clin Immunol 2020; 146: 831-839.e6.

31 Eddy RL, Svenningsen S, McCormack DG, et al. What is the minimal clinically important difference for helium-3 magnetic resonance imaging ventilation defects? Eur Respir J 2018; 51: 1800324.

32 He M, Driehuys B, Que LG, et al. Using hyperpolarized 129 Xe MRI to quantify the pulmonary ventilation distribution. Acad Radiol 2016; 23: 1521-1531. 
33 Serajeddini H, Eddy RL, Licskai C, et al. FEV1 and MRI ventilation defect reversibility in asthma and COPD. Eur Respir J 2019; 55: 1901947.

34 Bannier E, Cieslar K, Mosbah K, et al. Hyperpolarized ${ }^{3} \mathrm{He}$ MR for sensitive imaging of ventilation function and treatment efficiency in young cystic fibrosis patients with normal lung function. Radiology 2010; 255: 225-232.

35 Marshall H, Horsley A, Taylor CJ, et al. Detection of early subclinical lung disease in children with cystic fibrosis by lung ventilation imaging with hyperpolarised gas MRI. Thorax 2017; 72: 760-762.

36 Altes TA, Johnson M, Fidler M, et al. Use of hyperpolarized helium-3 MRI to assess response to ivacaftor treatment in patients with cystic fibrosis. J Cyst Fibros 2017; 16: 267-274.

37 Graham BL, Steenbruggen I, Barjaktarevic IZ, et al. Standardization of spirometry 2019 Update: An Official American Thoracic Society and European Respiratory Society Technical Statement. Am J Respir Crit Care Med 2019; 200: E70-E88. 